\title{
Super-Resolution Model for a Compressed-Sensing Measurement Setup
}

\author{
Torsten Edeler, Kevin Ohliger, Stephan Hussmann, Senior Member, IEEE, and \\ Alfred Mertins, Senior Member, IEEE
}

\begin{abstract}
In this paper, we present a new compressed-sensing (CS) setup together with a new scalable CS model, which allows the tradeoff between system complexity (number of detectors) and time (number of measurements). We describe the calibration of the system with respect to model parameters and show the reconstruction of compressed measurements according to the new model, which are acquired with the proposed setup. The proposed model and its parameter are evaluated with the established measures, i.e., restricted isometry property and coherence. The resulting consequences for usable sparsifying basis are derived on this evaluation. With the proposed setup, it is possible to acquire high-resolution images with a low-resolution camera.
\end{abstract}

Index Terms-Calibration, compressed sensing (CS), deblurring, deconvolution, single-pixel camera, super resolution, wavelet.

\section{INTRODUCTION}

$\mathbf{I}$ N RECENT years, a theory named Compressive Sensing (CS) has emerged, which overcomes the limitations that Shannon's theorem imposes on sampling systems. CS is motivated by the fact that most natural signals are sparse or at least approximately sparse in a certain basis such as a wavelet or Fourier basis. CS exploits the signal compressibility during the sampling process by measuring a few informative signal parts directly and therefore makes it possible to reduce the sampling rate drastically.

Assume a signal vector $\mathbf{x} \in \mathbb{R}^{N}$ has a $K$-sparse representation $\mathbf{x}=\boldsymbol{\Psi} \mathbf{c}$, for $\mathbf{c} \in\left\{\boldsymbol{\xi} \in \mathbb{R}^{N}:\|\boldsymbol{\xi}\|_{0} \leq K\right\}$, in representation basis $\left[\boldsymbol{\psi}_{n}\right]_{1}^{N}=\boldsymbol{\Psi}$. Sparsifying matrix $\tilde{\boldsymbol{\Psi}}$ is thereby the inverse of quadratic matrix $\Psi$. Sampling $M$ projections of $\mathrm{x}$ leads to the well-known CS model

$$
\mathbf{y}=\left[\left\langle\phi_{m}, \mathbf{x}\right\rangle\right]_{M}^{1}+\mathbf{v}=\mathbf{\Phi} \mathbf{x}+\mathbf{v}=\boldsymbol{\Phi} \Psi \mathbf{c}+\mathbf{v}
$$

where $\boldsymbol{\Phi}$ is the system sensing matrix as it represents the behavior of the measurement system. Together with the

Manuscript received June 20, 2011; revised August 15, 2011; accepted August 17, 2011. Date of publication December 1, 2011; date of current version April 6, 2012. This work was supported in part by the European Union under European Funds for Regional Development (EFRE) and in part by the Federal State of Schleswig-Holstein, Germany, under Zukunftsprogramm Wirtschaft. The Associate Editor coordinating the review process for this paper was Dr. Domenico Grimaldi.

T. Edeler, K. Ohliger, and S. Hussmann are with the Institute Ma.Vi.Tec, Westcoast University of Applied Sciences, 25746 Heide, Germany (e-mail: edeler@fh-westkueste.de).

A. Mertins is with the Institute for Signal Processing, University of Lübeck, 23562 Lübeck, Germany.

Color versions of one or more of the figures in this paper are available online at http://ieeexplore.iee.org.

Digital Object Identifier 10.1109/TIM.2011.2174891 representation matrix, they form the sampling matrix $\Phi \Psi$ for vector $\mathbf{c}$, which is the $K$-sparse representation of x. Observation noise is taken into account by $\mathbf{v}$. The CS theory tells us that, in certain situations, there exists a unique solution to the inverse problem of finding $\mathbf{c}$ given $M<N$ measurements.

The concept of CS has been successfully applied to realworld applications known as single-pixel cameras [1]-[3], which have only one detector element instead of millions to capture an image. This is useful in applications where either due to cost or size (large) arrays of detectors are not practical.

The drawback of existing concepts is the limitation to one detector. We propose a scalable model that allows detector arrays of arbitrary size, with the single-pixel camera being a special case of array-size one. This model allows for the tradeoff between number of measurements (time) and system complexity (number of detectors).

Using multiple detectors in the CS context is not new and has been used in [4] to reconstruct a single source image by assuming sparse representation of single image tiles using overcomplete dictionaries. In [5], the authors used several audio receivers to reconstruct a source signal. They assume the measurements to be a shifted and filtered version of the original signal. In [6], the authors present a general framework for related but not identical signals supplied by several detectors. They introduce a structured sparsity model and assume the detector signals to be represented by such a model. In contrast to other works, we propose a model and reconstruction method that does not assume a shared sparse representation or structure of the individual detector signals but a sparse representation of the whole source signal. This is, for example, important in cases where an image is sampled by multiple detectors, which only cover small parts of the whole image.

The problem of multidetector compressed measurements, where each detector has a different spatial intensity, has been also proposed in [7] for the MRI context, where multiple coils directly sample in Fourier space. In contrast to our work, MRI measurements are performed in a transformed domain but the coils have different spatial responsivities. Although our model also describes different spatial responsivities, it also does the sampling in a spatial domain, which is different to MRI measurements.

In [8], the authors present a multidetector compressed measurement model, which is essential to a parallel single-pixel camera. Each of those cameras reconstructs a small part of the full image. This approach is very similar to that presented in this paper. However, one major difference is that our model does not assume independence of the parallel single-pixel cameras. 
(a)

(d)

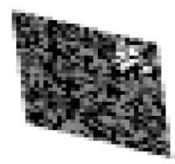

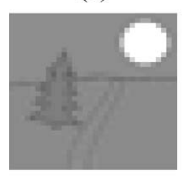

(b)

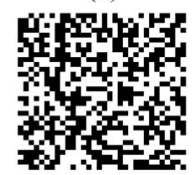

(e)

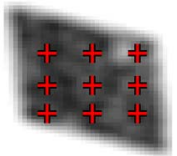

(c)

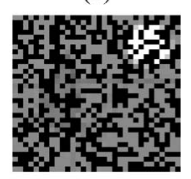

(f)

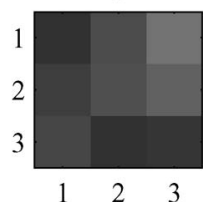

Fig. 1. Visualization of model (2). (a) High-resolution image x. (b) Random weighting pattern $\operatorname{diag}\left[\mathbf{F}_{k}\right]$. (c) Weighted original image $\mathbf{F}_{k} \mathbf{x}$. (d) Projective sampled image $\mathbf{D F}_{k} \mathbf{x}$. (e) Blurred image $\mathbf{H D F}_{k} \mathbf{x}$ with sampling locations of operator $\mathbf{P}$ marked with crosses. (f) Measurement result $\mathbf{y}_{k}$ with $N_{x} \cdot N_{y}=9$ detectors.

Our model covers the acquisition of $N_{x} \cdot N_{y}$ different (but possibly similar) linear mixtures of an original image

$$
\mathbf{y}_{k}=\mathbf{P H D F}_{k} \mathbf{x}+\mathbf{v}_{k} \quad \forall k \in\{1 \ldots M\}
$$

where $\mathbf{x} \in \mathbb{R}^{\left[N_{u} N_{v} \times 1\right]}$ is the (lexicographically ordered) highresolution image, and $\mathbf{y}_{k} \in \mathbb{R}^{\left[N_{x} N_{y} \times 1\right]}$ is the $k$ th measurement of the mixtures. Matrix $\mathbf{F}_{k}$ is diagonal and contains the weights for each element in $\mathbf{x}$. In Fig. 1, this model is visualized. The original image $\mathbf{x}$ [see Fig. 1(a)] is element wise multiplied by pattern $\operatorname{diag}\left[\mathbf{F}_{k}\right]$ [see Fig. 1(b)]. The result [see Fig. 1(c)] is projected onto the detector array by operator $\mathbf{D}$ [see Fig. 1(d)], blurred by convolution operator $\mathbf{H}$ [see Fig. 1(e)], and sampled on a regular rectangular grid by operator $\mathbf{P}$ [see Fig. 1(e)]. The result $\mathbf{y}_{k}$ with $N_{x} \cdot N_{y}=9$ elements is shown in Fig. 1(e).

Reconstructing $\mathbf{x}$ from measurements $\mathbf{y}_{k}$ with the prior knowledge that $\mathrm{x}$ has a sparse representation $\mathrm{c}=\tilde{\Psi}_{\mathrm{x}}$ can be stated as

$$
\hat{\mathbf{x}}=\arg \min _{\mathbf{x}} \sum_{k=1}^{M}\left\|\mathbf{y}_{k}-\mathbf{P} \mathbf{H D F} \mathbf{F}_{k} \mathbf{x}\right\|_{2}^{2} \quad \text { s.t. } \quad\|\tilde{\mathbf{\Psi}} \mathbf{x}\|_{0}=K
$$

where $K$ is the given sparsity. It is known from literature that this problem is in principle combinatorial and NP hard to solve [9]. However, under certain conditions [10], (3) can be relaxed to

$$
\hat{\mathbf{x}}=\arg \min _{\mathbf{x}}\|\tilde{\mathbf{\Psi}} \hat{\mathbf{x}}\|_{1} \quad \text { s.t. } \quad \sum_{k=1}^{M}\left\|\mathbf{y}_{k}-\mathbf{P H D F}_{k} \hat{\mathbf{x}}\right\|_{2}^{2} \leq \epsilon
$$

where $\epsilon$ is the expected error because of measurement noise or not exactly sparse signal $\mathbf{x}$. The conditions under which (3) and (4) yield the same result are noiseless measurement, exact sparse representation $(\epsilon=0)$, and certain quality criteria given in Section IV.

Note that model (2) is equal to a single measurement of model (1) if PHD $=[1]_{N_{u} \cdot N_{v}}^{1}$. In this case, our model describes a single detector $\left(N_{x}=N_{y}=1\right)$, and the proposed reconstruction method is equal to the one proposed in [11].

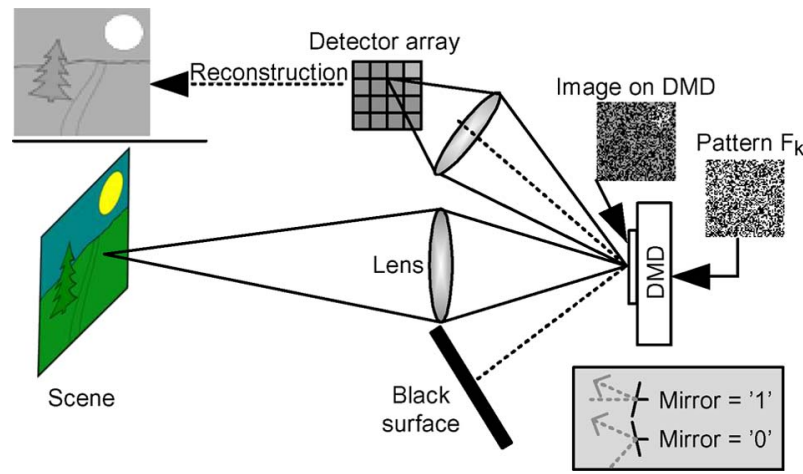

Fig. 2. Schematic of test setup. A scene is projected by a lens on reference plane $\Gamma$ where a DMD lies. The DMD optically multiplies the projected scene by a binary pattern by switching mirrors to the scene or to a black surface. The multiplication result is then projected by a second lens onto image plane $\Pi$ where a low-resolution detector array is located. Using the CS theory, a highresolution image can be reconstructed with this setup.

In [12], the authors present a CS deblurring method, which is similar to ours. The difference of our method is that we consider geometric transformations $(\mathbf{P}, \mathbf{D})$. We also have control over the inner product that is influenced by the element-wise multiplication, represented by diagonal matrices $\mathbf{F}_{k}$.

In [13], we presented the first research results of the proposed system. In this paper, we have extended the reference list, the theoretical, and the experimental sections of the conference proceeding paper. Established quality measures are newly introduced in Section IV, and we use them to show the performance of our model with respect to the number of measurements and to give a statement on good sparsifying basis for reconstruction. The experiments are extended by simulations and reconstructions from real measurements.

This paper is structured as follows: We describe a real measurement setup in Section II that fits to model (2), and in Section III, we show how to calibrate the model parameters to that setup. The quality of measurement systems that can be described by our model is given in Section IV for different model parameters. Measurements of the estimated parameters are presented in Section V together with the application of super-resolution reconstruction of simulated and real compressive measurements.

\section{Measurement Setup}

To evaluate model (2) in practice, we build up a test setup, which is shown as a schematic in Fig. 2. The main components of the setup are the digital mirror device (DMD) as the multiplication element to implement matrix $\mathbf{F}_{k}$ and an area camera as the detector array. The observed scene is projected by a first lens onto the DMD, which, in turn, is projected with a second lens onto the detector array. The DMD consists of thousands of switchable mirrors, which either direct the light from the scene or from a black surface to the detector array and thus is able to "multiply" the scene image by a pattern.

The center of each mirror on the DMD lies in the reference plane $\boldsymbol{\Gamma}$ at location $\mathbf{x}^{\prime}=\left[u \cdot \Delta_{\gamma, u}, v \cdot \Delta_{\gamma, v}\right]^{T}$, with $u \in\{n \in$ $\left.\mathbb{N}: n \leq N_{v}\right\}$ and $v \in\left\{n \in \mathbb{N}: n \leq N_{u}\right\}$ as coordinates and $\left[\Delta_{\gamma, u} \times \Delta_{\gamma, v}\right]$ being the dimension of each mirror. Therefore, the resolution of the mirror array is $\left[N_{u} \times N_{v}\right]$. 
The detector array, which lies in image plane $\Pi$, captures the images $\mathbf{y}$ of the mirrors on plane $\boldsymbol{\Gamma}$. Each detector element is centered at location $\mathbf{x}=\left[x \cdot \Delta_{\pi, x}, y \cdot \Delta_{\pi, y}\right]^{T}$, with $x \in \Omega_{x}=$ $\left\{n \in \mathbb{N}: n \leq N_{x}\right\}$ and $y \in \Omega_{y}=\left\{n \in \mathbb{N}: n \leq N_{y}\right\}$ as its coordinates and $\left[\Delta_{\pi, x} \times \Delta_{\pi, y}\right]$ being the dimension of each detector. The detector array has therefore a resolution of $\left[N_{x} N_{y}\right]$ detectors. The intensity signal of the detector array is then

$$
I(x, y) \propto \int_{\Delta_{h} / 2}^{\Delta_{h} / 2} \int_{2} h\left(\tau_{x}, \tau_{y}\right) \cdot f\left(x-\tau_{x}, y-\tau_{y}\right) d \tau_{y} d \tau_{x}
$$

where function $f(\cdot, \cdot): \mathbb{R}^{2} \rightarrow \mathbb{R}$ represents the light intensity on image plane $\Pi, h(\cdot, \cdot): \mathbb{R}^{2} \rightarrow \mathbb{R}$ is the continuous blurring kernel, and $\Delta_{h}$ is its support in both dimensions. Note that $\Delta_{h} \geq\left\{\Delta_{\pi, x}, \Delta_{\pi, y}\right\}$. By lexicographic ordering of function $I(\cdot, \cdot):\left(\Omega_{x}, \Omega_{y}\right) \rightarrow \mathbb{R}$, it can be written as vector $\mathbf{y}$.

\section{System Calibration}

Model (2) consists of unknown operators $\mathbf{P}, \mathbf{H}$, and $\mathbf{D}$, which are determined by calibration. Downsampling $\mathbf{P}$ and projection $\mathbf{D}$ are determined by geometric calibration (GC), and convolution operator $\mathbf{H}$ is then estimated.

\section{A. $G C$}

For GC, the geometric relation between detectors and mirrors is determined. The center $\mathbf{x}_{i}^{\prime}$ of the $i$ th mirror in plane $\boldsymbol{\Gamma}$ is projected onto the image plane $\Pi$ at location $\mathbf{x}$. The relationship between $\mathrm{x}^{\prime}$ and $\mathrm{x}$ is given by homography [14] $\mathbf{T}$ with

$$
\left[\begin{array}{c}
\mathbf{x} \\
1
\end{array}\right] \propto \mathbf{T}\left[\begin{array}{c}
\mathbf{x}^{\prime} \\
1
\end{array}\right]=\left[\begin{array}{ccc}
t_{1,1} & t_{1,2} & t_{1,3} \\
t_{2,1} & t_{2,2} & t_{2,3} \\
t_{3,1} & t_{3,2} & 1
\end{array}\right]\left[\begin{array}{c}
\mathbf{x}^{\prime} \\
1
\end{array}\right] .
$$

Geometric system calibration is therefore the task of finding the eight unknowns in $\mathbf{T}$. This is commonly performed by taking known correspondence pairs $\left(\mathbf{x}_{k}^{\prime}, \mathbf{x}_{k}\right)$ and solving the least squares problem

$$
\hat{\mathbf{T}}=\arg \min _{\mathbf{T}} \sum_{k=1}^{N}\left\|\mathbf{x}_{k}^{\prime}-\hat{\mathbf{x}}_{k}\right\|_{2} \quad \text { with } \quad\left[\begin{array}{c}
\hat{\mathbf{x}} \\
1
\end{array}\right] \propto \mathbf{T}\left[\begin{array}{c}
\mathbf{x}^{\prime} \\
1
\end{array}\right]
$$

where $N$ is the number of known correspondence pairs.

Once $\hat{\mathbf{T}}$ is estimated, sampling operators $\mathbf{P}$ and $\mathbf{D}$ have to be defined. Each of them is associated with transformation matrices $\hat{\mathbf{T}}_{P}$ and $\hat{\mathbf{T}}_{D}$, respectively. The source image (right operand) is sampled on locations that correspond to integer locations in the destination image. Operators $\mathbf{P}$ and $\mathbf{D}$ use nearest neighbor and bilinear interpolation, respectively. The matrices fulfill condition $\hat{\mathbf{T}}=\hat{\mathbf{T}}_{P} \hat{\mathbf{T}}_{D}$, and $\hat{\mathbf{T}}_{D}$ is chosen the way that the smallest projected mirror has no side length smaller than four on the image plane. Matrix $\hat{\mathbf{T}}_{P}$ is defined as

$$
\hat{\mathbf{T}}_{P}=\left[\begin{array}{ccc}
S_{1} & 0 & \hat{t}_{1,3} \\
0 & S_{2} & \hat{t}_{2,3} \\
0 & 0 & 1
\end{array}\right]
$$

where $\hat{t}_{i, j}$ are the elements of $\hat{\mathbf{T}}$, and $S_{1,2}$ are the scale factors for the grid implicit to $\hat{\mathbf{T}}_{P}$ [see Fig. 1(e)].

For finding pairs $\left(\mathbf{x}_{k}^{\prime}, \mathbf{x}_{k}\right)$, we use $N$ not connected circular control points loaded as pattern on the homogeneously illuminated DMD. Each control points with radius $r$ is represented by a region $\mathcal{R}_{k}^{\prime}$ on the mirror array. The $i$ th mirror at location $\mathbf{x}_{i}^{\prime}$ is set towards the scene $\left(M_{i}=1\right)$, if it belongs to one of the regions and is set towards the black surface $\left(M_{i}=0\right)$ if not:

$$
M_{i}=\left\{\begin{array}{cc}
1 & \mathbf{x}_{i}^{\prime} \in \mathcal{R}_{k}^{\prime} \\
0 & \mathbf{x}_{i}^{\prime} \notin \mathcal{R}_{k}^{\prime}
\end{array}= \begin{cases}1 & {\left[\begin{array}{c}
\mathbf{x}_{i}^{\prime} \\
1
\end{array}\right]^{T} \mathbf{Q}_{k}\left[\begin{array}{c}
\mathbf{x}_{i}^{\prime} \\
1
\end{array}\right] \leq 0} \\
0 & {\left[\begin{array}{c}
\mathbf{x}_{i}^{\prime} \\
1
\end{array}\right]^{T} \mathbf{Q}_{k}\left[\begin{array}{c}
\mathbf{x}_{i}^{\prime} \\
1
\end{array}\right]>0}\end{cases}\right.
$$

where $\mathcal{R}_{k}^{\prime}$ is the region of the $k$ th control point with $k \in\{n \in$ $\mathbb{N}: n<N\}$, and $M_{i} \in\{0,1\}$ is the state of the $i$ th mirror. Radius $r$ and center location $\mathbf{x}_{0}^{\prime}=\left[u_{0}, v_{0}\right]$ of the $k$ th control point are defined by conic section $\mathbf{Q}_{k}$, which, in the case of a circular shape with radius $r$, has the form

$$
\mathbf{Q}_{k}=\left[\begin{array}{ccc}
1 & 0 & -u_{0} \\
0 & 1 & -v_{0} \\
-u_{0} & -v_{0} & u_{0}^{2}+v_{0}^{2}-r^{2}
\end{array}\right] .
$$

All $N$ regions $\mathcal{R}_{k}^{\prime}$, displayed on the DMD, are projected onto the image plane and form regions $\mathcal{R}_{k}$ of connected elements in observed images $\mathbf{y}$. The center points $\mathbf{x}_{k}^{\prime}$ and $\mathbf{x}_{k}$ of both regions are used as pairs to solve (7).

When detecting the connected regions in the image $\mathbf{y}$, the problem of mapping a region to the correct source region $k$ remains. We solve this by taking several images $\mathbf{y}_{f}$ and hide or display each region $\mathcal{R}_{k}^{\prime}$ on the DMD according to an associated pattern $\mathbf{p}_{k}=\left[p_{k}^{1} \ldots p_{k}^{M}\right] \in\{0,1\}$, which fulfills condition $k=\sum_{m=1}^{M} 2^{m-1} p_{m}^{k}$, with $M=\left\lceil\log _{2}(N+1)\right\rceil$. Using this procedure, each observed region can be associated with the corresponding source region $\mathcal{R}_{k}^{\prime}$ on the DMD.

\section{B. Circular Control Points Under Geometric Projection}

In general, projection is not shape preserving. This is especially true if circular control points are used for a point-to-point correspondence between the reference and image planes. A bias is introduced if the centers of the projected circles on the image plane are treated as the projected circle centers.

A conic section is a quadratic curve and defined by a matrix $\mathbf{Q}$. Locations $\mathrm{x} \in \mathbb{R}^{2}$ on a plane are on the curve if they fulfill the condition

$$
0=\left[\begin{array}{l}
\mathbf{x} \\
1
\end{array}\right]^{T} \mathbf{Q}\left[\begin{array}{c}
\mathbf{x} \\
1
\end{array}\right] .
$$

The center $\mathbf{x}_{0}$ of conic section $\mathbf{Q}$ is defined with the pole of the line at infinity $\mathbf{L}_{\infty}=[0,0,1]^{T}$

$$
\left[\begin{array}{c}
\mathbf{x}_{0} \\
1
\end{array}\right] \propto \mathbf{Q}^{-1} \mathbf{L}_{\infty}=\mathbf{Q}^{-1}\left[\begin{array}{l}
0 \\
0 \\
1
\end{array}\right] \text {. }
$$

The regions $\mathcal{R}_{k}^{\prime}$ on reference plane $\Gamma$ enclosed by a conic section $\mathbf{Q}_{k}$ with center point $\mathbf{x}_{k, 0}^{\prime}$ are projected onto image plane $\Pi$ to regions $\mathcal{R}_{k}$ enclosed by conic section $\mathbf{B}_{k} \propto$ 
$\left(\mathbf{T}^{-1}\right)^{T} \mathbf{Q}_{k} \mathbf{T}^{-1}$ with center point $\mathbf{x}_{k, 0}$. In general, the center point $\mathbf{x}_{k, 0}$ of $\mathbf{B}_{k}$ on the image plane is not the projection of the center point $\mathbf{x}_{k, 0}^{\prime}$ of $\mathbf{Q}_{k}$ from the reference plane. Instead, $\mathbf{x}_{k, 0}^{\prime}$ is projected to

$$
\left[\begin{array}{c}
\hat{\mathbf{x}}_{k, 0} \\
1
\end{array}\right] \propto \mathbf{T}\left[\begin{array}{c}
\mathbf{x}_{k, 0}^{\prime} \\
1
\end{array}\right] \propto \mathbf{T Q}_{k}^{-1}\left[\begin{array}{l}
0 \\
0 \\
1
\end{array}\right]
$$

on the image plane. On the other hand, the center point of $\mathbf{B}_{k}$ is

$$
\left[\begin{array}{c}
\mathbf{x}_{k, 0} \\
1
\end{array}\right] \propto \mathbf{B}^{-1}\left[\begin{array}{l}
0 \\
0 \\
1
\end{array}\right] \propto \mathbf{T Q}_{k}^{-1} \mathbf{T}^{T}\left[\begin{array}{l}
0 \\
0 \\
1
\end{array}\right]=\mathbf{T} \mathbf{Q}_{k}^{-1}\left[\begin{array}{c}
t_{3,1} \\
t_{3,2} \\
1
\end{array}\right]
$$

The circle $\mathbf{Q}_{k}$, with $\mathbf{x}_{k, 0}^{\prime}$ as the center point, on reference plane $\boldsymbol{\Gamma}$ is projected to an ellipse $\mathbf{B}_{k}$, with $\mathbf{x}_{k, 0}$ as the center point, on image plane $\Pi$. In performing a GC using point-to-point correspondence, it is necessary to know point $\hat{\mathbf{x}}_{k, 0}$, which is the projection of the circle's center point on plane $\Pi$. In the case of affine projection $\left(t_{3,1}=t_{3,2}=0\right)$, (14) shows that $\hat{\mathbf{x}}_{k, 0}=\mathbf{x}_{k, 0}$ and our calibration procedure, as described above, is correct. In our setup, we have very fine control over the DMD orientation and we can tune the position to have affine projection. In the case of projective transformation, [15] describes a method for finding the elements in $\mathbf{T}$ by solving a least squares problem using the relationship in (14).

\section{Calibration of Blurring Operator}

Here, we describe our method to estimate the blurring kernel in model (2). The 2-D discrete convolution is defined by

$$
y(u, v)=\sum_{j=-k}^{k} \sum_{i=-k}^{k} x(u-j, v-i) \cdot h(j, i)
$$

where $x, y: \mathbb{Z}^{2} \rightarrow \mathbb{R}$ are the source and convolved source images, respectively, and $h:\{n \in \mathbb{Z}:-k \leq n \leq k\}^{2} \rightarrow \mathbb{R}$ is the impulse response of the convolution filter. If $x(u, v)=0$ for all $u \notin \Omega_{1}$ and $v \notin \Omega_{2}$, with $\Omega_{i}=\left\{n \in \mathbb{N}: n \leq N_{i}\right\}$, it can be represented by a vector $\mathbf{x} \in \mathbb{R}^{N_{1} N_{2} \times 1}$. Moreover, if (15) is only evaluated on locations $u \in \Omega_{1}$ and $v \in \Omega_{2}$, it can be written as

$$
\mathbf{y}=\sum_{j=-k}^{k} \sum_{i=-k}^{k} \mathbf{S}_{1}^{j} \mathbf{S}_{2}^{i} \mathbf{x} \cdot h(-j,-i)=\mathbf{X} \mathbf{h}=\mathbf{H x}
$$

where $\mathbf{y} \in \mathbb{R}^{N_{1} N_{2} \times 1}$ is the convolution result, and matrices $\mathbf{S}_{u}^{j}$ and $\mathbf{S}_{v}^{i}$ are the shift operators. If vector $\mathbf{x}$ is associated with function $x(u, v)$, vector $\mathbf{S}_{1}^{j} \mathbf{S}_{2}^{i} \mathbf{x}$ is associated with function $x(u+i, v+j)$. The columns of $\mathbf{X}$ contain all $(2 k+1)^{2}$ shifted versions of $\mathbf{x}$, and $\mathbf{h} \in \mathbb{R}^{(2 k+1)^{2} \times 1}$ is the lexicographically ordered vector representation of function $h(\cdot, \cdot)$. Operator $\mathbf{H}$ is the convolution matrix in (2) with blurring kernel $h(\cdot, \cdot)$. Combining model (2) with (16) yields

$$
\mathbf{y}_{k}=\mathbf{P} \mathbf{H D F}_{k} \mathbf{x}+\mathbf{v}_{k}=\mathbf{P H z}+\mathbf{v}_{k}=\mathbf{P Z h}+\mathbf{v}_{k}
$$

where $\mathbf{z}=\mathbf{D F}_{k} \mathbf{x}$. In the setup, $\mathbf{D}$ and $\mathbf{P}$ are known from the calibration process, and matrix $\mathbf{F}_{k}$ depends on the arbitrary binary pattern loaded into the DMD. The columns of $\mathbf{Z}$ are shifted versions of $\mathbf{z}$. During calibration, the DMD is uniformly illuminated, which allows setting $\mathbf{x}=\xi \cdot[1]_{N}^{1}$, where $\xi$ is the illumination intensity. Equation (17) can be then solved with respect to the impulse response $\mathbf{h}$ in the least squares sense by

$$
\mathbf{h}=(\mathbf{P Z})^{\dagger} \mathbf{y}_{k}
$$

where $\mathbf{A}^{\dagger}$ denotes the pseudoinverse of $\mathbf{A}$.

\section{Quality Measures for Measurement Matrices AND TheIR IMPLICATIONS FOR THE TEST SETUP}

In the last section, we discussed how to calibrate our proposed model with a given setup. Here, we concentrate on the quality of that model. First, we introduce two popular and well-established measures for CS, namely, restricted isometry property (RIP) and coherence. Then, we test different model parameters against these measures and derive consequences for the measurement setup configuration and the reconstruction strategy.

\section{A. RIP}

The RIP is a matrix property introduced by Candès and Tao in [11], where it is called the uniform uncertainty principle. The original symmetric RIP for a matrix $\mathbf{A}=\left[\mathbf{a}_{i}\right]_{1}^{N} \in\left\{\mathbb{R}^{P \times N}\right.$ : $\left.\left\|a_{i}\right\|_{2}=1\right\}$ is defined as

$$
\left(1-\delta_{r}\right) \leq\|\boldsymbol{A} \mathbf{c}\|_{2}^{2} \leq\left(1+\delta_{r}\right)
$$

where $\mathbf{c} \in\left\{\mathbb{R}^{N}:\|c\|_{2}=1,\|c\|_{0}=r\right\}$ is an $r$-sparse vector, and $\delta_{r}$, the restricted isometry constant (RIC), is the smallest value given all possible vectors $\mathbf{c}$ that fulfill condition (19). Alternatively, the RIC can be understood as being the maximum distance from 1 for all eigenvectors of all possible matrices $\mathbf{A}_{\mathcal{I}} \mathbf{A}_{\mathcal{I}}^{T}$, where $\mathbf{A}_{\mathcal{I}}=\left[\mathbf{a}_{i}\right]_{\mathcal{I}}$, and $\mathcal{I}$ is a set of indices with cardinality $|\mathcal{I}|=s$. It can be easily shown that an RIC with $\delta_{2 s}<1(r=2 s)$ allows identification of all possible $s$-sparse vectors, where identification means that there are no two $s$ sparse vectors that are projected by $\mathbf{A}$ to the same point [11]. In [16], it is also shown that the convex relaxation (4) of (3) is exact if $\delta_{2 s} \leq \sqrt{2}-1$, and the authors also give an upper reconstruction error bound for the case where the original nonsparse signal is reconstructed from noisy measurements.

The RIP has been proven to be a useful tool in analyzing reconstruction methods and decoders in the CS context (see [17] and [11]). Due to its combinatorial nature, it is not practical to calculate the actual value of the RIP constants for a given sampling matrix. In fact, determining the RIP constants of order $s$ for a matrix of size $[P \times N]$ requires one to calculate minimum and maximum singular values of all possible $\left(\begin{array}{l}n \\ k\end{array}\right)$ submatrices. In most literature, random matrices are used because of known asymptotic properties. Other authors use Monte Carlo analysis [18] to avoid checking every possible submatrix. However, as stated in [18], this analysis is not able to find pathological submatrices and thus is, in most of the cases, too 
optimistic. Recently, a new greedy algorithm has been proposed in [19], which is able to find pathological submatrices while still being computationally cheap. With this algorithm, for the first time, it is possible to explicitly have a good estimation for the RIP constants for a given reasonable big matrix.

\section{B. Coherence and Transformed Point-Spread Function}

Mutual incoherence was first presented in the context of basis pursuit ( $\ell_{1}$ minimization) by Donoho and Huo in 2002 [20]. It measures the dissimilarity or incoherence between two bases $\boldsymbol{\Omega}_{1}=\left[\boldsymbol{\omega}_{i}^{1}\right]^{N}$ and $\boldsymbol{\Omega}_{2}=\left[\boldsymbol{\omega}_{i}^{2}\right]^{N}$ and is defined as

$$
\mu\left(\boldsymbol{\Omega}_{1}, \boldsymbol{\Omega}_{2}\right)=\max _{i, j}\left\langle\boldsymbol{\omega}_{i}^{1}, \boldsymbol{\omega}_{j}^{2}\right\rangle=\max _{i, j} U_{i, j}
$$

where $\mathbf{U}=\Omega_{1} \boldsymbol{\Omega}_{2}^{T}$ and $U_{i, j}$ are the elements of $\mathbf{U}$. The mutual coherence of the two orthonormal bases with

$$
\left.\left\|\boldsymbol{\omega}_{i}^{1,2}\right\|_{2}\right|_{i \in\{n \in \mathbb{N}: n \leq N\}}=1
$$

is obviously framed by $1 / \sqrt{N} \leq \mu\left(\boldsymbol{\Omega}_{1}, \boldsymbol{\Omega}_{2}\right) \leq 1$. A value of one means that both bases have at least one basis vector in common, and $1 / \sqrt{N}$ means maximal incoherence between the two bases. In the context of CS, mutual incoherence is of interest as we have system sensing matrix $\boldsymbol{\Phi}$ and representation matrix $\Psi$, which are presented in model (1). For successful $\mathrm{CS}$, both matrices should be as incoherent as possible, which is equivalent to the statement that concentrated energy in representation space is spread out in measurement space.

In addition to the presented mutual incoherence, there exists a similar definition of coherence in [21], which is strongly related to the transformed point-spread function (TPSF) introduced in [7]. Given a sampling system $\mathbf{y}=\mathbf{A x}$ where $\mathbf{x}$ is sparse, the quality of sampling matrix $\mathbf{A}$ with respect to sparse reconstruction can be determined by its TPSF, which is defined by

$$
\operatorname{TPSF}(i, j)=\mathbf{e}^{i} \mathbf{A}^{T} \mathbf{A} \mathbf{e}^{j}
$$

where $\mathbf{e}^{k}$ is a unit vector with the $k$ th element set to one and the rest to zero. The TPSF in matrix notation is equal to Gram matrix $\mathbf{G}=\mathbf{A}^{T} \mathbf{A}=\left[\mathbf{g}_{j}\right]_{j=1}^{N}$, where $\mathbf{g}_{j}=\left[g_{j}^{i}\right]_{j=1}^{N}$ are column vectors of size $N$. Coherence $\mu(\mathbf{A})$, as defined in [21], is then

$$
\mu(\mathbf{A})=\max _{j \neq i}\left|\frac{g_{j}^{i}}{g_{i}^{i}}\right|
$$

In contrast to the RIP, the TPSF and the coherence are very easy to calculate as there is no combinatorial problem involved. Moreover, as well as RIP, $\mu(\cdot)$ provides performance guaranties with respect to $\ell_{1}$ solutions. For example, in [21], it is shown that, if $\mathbf{x}$ is $K$-sparse and $K<(1 / 4)\left[\mu^{-1}(\mathbf{A})+1\right]$, then the $\ell_{1}$ solution of (23) is guaranteed to provide a squared reconstruction error less than or equal to $4 \epsilon^{2}(1-\mu(\mathbf{A})[4 K-1])^{-1}$. In [22], the authors showed a connection between the coherence and the RIP of a given matrix with respect to $K$-sparse signal reconstruction. They proved that $\delta_{k}(\mathbf{A}) \leq(K-1) \mu(\mathbf{A})$, which provides an easy-to-calculate upper bound for the RIC. (a)

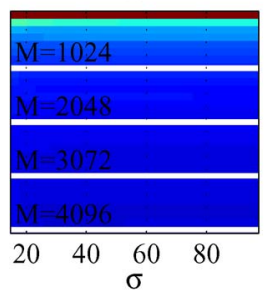

(c)

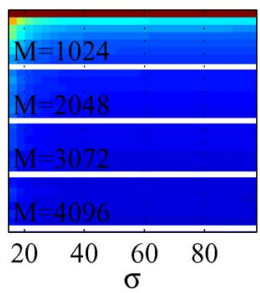

(b)

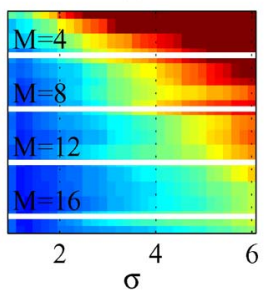

(d)

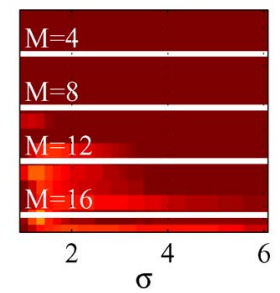

$\mu^{D C T}$

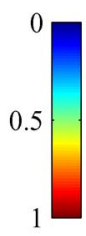

$\mu^{\text {Haar }}$

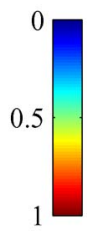

Fig. 3. Coherence $\mu$ of measurement matrices $A^{\mathrm{DCT}}$ and $A^{\text {Haar }}$ over model parameters $M$ and $\sigma$ for [(a) and (c)] single-detector and [(b) and (d)] multidetector setups. Lower values mean that better CS performance can be expected. For all cases, performance increases with increasing sequent number of measurements. (a) Single-detector setup $\left(l^{2}=1\right.$ detectors) for DCT basis. (b) Multidetector setup $\left(l^{2}=256\right.$ detectors) for DCT basis. (c) Single-detector setup $\left(l^{2}=1\right)$ for Haar wavelet basis. (d) Multidetector setup $\left(l^{2}=256\right)$ for Haar wavelet basis.

\section{Implication of Model Parameters on Reconstruction Quality}

Here, we study the implication of basic model parameters on the two measures introduced above. To keep things simple, we use a simplified model (2) with homography

$$
\mathbf{T}=\left[\begin{array}{ccc}
l \cdot N_{u}^{-1} & 0 & 0.5 \\
0 & l \cdot N_{u}^{-1} & 0.5 \\
0 & 0 & 1
\end{array}\right]
$$

where $l$ is the scaling factor. Blurring operator $\mathbf{H}$ is defined by a 2-D Gaussian symmetric blurring kernel with standard deviation $\sigma$ and support of $4 \sigma$ pixels in both dimensions. This simplified model implies no other distortion than equally distant downsampling and blurring, and it provides three degrees of freedom for sampling, i.e., 1) number of sequent measurements $M ; 2$ ) blurring intensity $\sigma$; and 3 ) downsampling factor $l$. With respect to reconstruction, we also have the freedom to chose representation basis $\boldsymbol{\Psi}$. Both coherence and RIP measure the quality with respect to matrix $\mathbf{A}$, which directly measures the sparse signal representation

$$
\mathbf{y}=\left[\begin{array}{c}
\mathbf{y}_{1} \\
\vdots \\
\mathbf{y}_{M}
\end{array}\right]=\left[\begin{array}{c}
\mathbf{P H D F}_{1} \\
\vdots \\
\mathbf{P H D F}_{M}
\end{array}\right] \mathbf{\Psi}^{B} \mathbf{c}=\mathbf{S} \boldsymbol{\Psi}^{B} \mathbf{c}=\mathbf{A}^{B} \mathbf{c}
$$

where $\mathbf{S}$ is the sampling matrix, and $\mathbf{A}^{B}$ includes the complete sampling process, including all $M$ sequent measurements in representation basis $\Psi^{B}$. In our quality experiments, we use the discrete cosine transform $(B=\mathrm{DCT})$ and the Haar wavelet transform ( $B=$ Haar) as bases.

In Figs. 3 and 4, we measured the coherence $\mu^{B}$ and the symmetric RIC $\delta^{B}$ for two scaling factors $(l=1,16)$ 
(a)

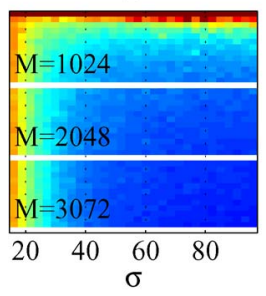

(c)

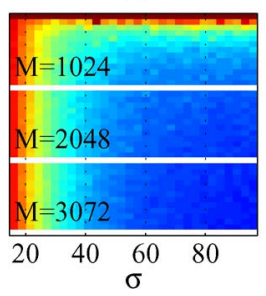

(b)

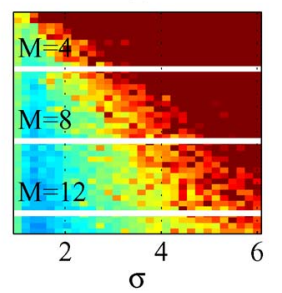

(d)

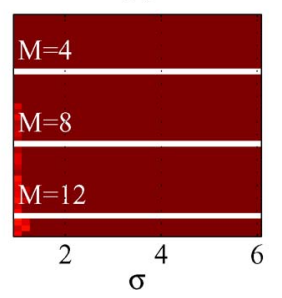

$\delta_{10}^{D C T}$

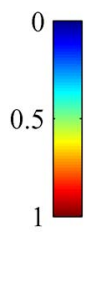

$\delta_{10}^{\text {Haar }}$

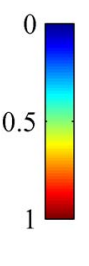

Fig. 4. RIC $\delta_{10}$ of measurement matrices $A^{\mathrm{DCT}}$ and $A^{\text {Haar }}$ over model parameters $M$ and $\sigma$ for [(a) and (c)] single-detector and [(b) and (d)] multidetector setups. Lower values mean that better CS performance can be expected. For values of $\delta_{10} \leq \sqrt{2}-1 \approx 1.4$, perfect reconstruction of an exactly $(K=5)$ sparse signal is possible using (4). (a) Single-detector setup $\left(l^{2}=1\right.$ detectors) for DCT basis. (b) Multidetector setup $\left(l^{2}=16\right.$ detectors) for DCT basis. (c) Single-detector setup $\left(l^{2}=1\right)$ for Haar wavelet basis. (d) Multidetector setup $\left(l^{2}=16\right)$ for Haar wavelet basis.

over the number of sequent measurements $M$ and blurring intensity $\sigma$ for the DCT $(B=$ DCT $)$ and Haar bases $(B=$ Haar). The high-resolution image was set to $\left[N_{u} \times N_{v}\right]=$ $[32 \times 32]$ pixels.

The first thing to notice is that both coherence and RIP measures provide qualitative comparable results. To the best of our knowledge, this is the first time both measures are compared over parametric scans.

In the case of $l=1$ and high values for $\sigma$, we have a model configuration that is similar or equal to the single-pixel camera because the single-detector element then integrates over the whole high-resolution image [see (5)]. As known from literature, with a rising number of sequent measurements $M$, the quality of the sampling matrix increases when using a single-detector element. This is indicated by low values of $\mu^{B}$ and $\delta_{s}^{B}$ in Figs. 3(a) and (c) and 4(a) and (c). In contrast, when using multiple detectors, DCT or Haar basis differs with respect to $\mu^{B}$ and $\delta_{s}^{B}$. According to Figs. 3(b) and (d) and 4(b) and (d), more measurements only improve the quality if DCT is used as the sparsifying basis. The reason for this is the high mutual coherence between the Haar basis $\boldsymbol{\Psi}^{\text {Haar }}$ and the system sensing matrix $\mathbf{S}$ from (25). This is easy to see because, when using a small blurring kernel and multiple detectors, each single-detector elements does not "see" the entire high-resolution image. The Haar basis (as every wavelet basis) encodes frequency and local information, and because of this, it is possible for a signal that is sparse in the wavelet basis to be not detectable by the single pixel, which does not cover the whole high-resolution image. This is the reason for the degraded performance with small values of $\sigma$. In contrast to this, the DCT basis does not encode local but only frequency information. Consequently, a signal that is sparse in the DCT basis has its energy spread all over and it is more likely to be detected by a single detector, which does not cover the whole high-resolution image.

Note that the performance measures improve with increasing $M$. This is because matrix $\mathbf{A}^{B}$ has dimension $\left[M l^{2} \times N_{u} N_{v}\right]$ and the problem gets less ill posed.

When comparing the single-pixel model $(l=1)$ with the multipixel model $(l=16)$ in the DCT basis, it is obvious that the number of sequent measurements $(M)$ can be dramatically reduced when using multiple detectors. For example, with a single pixel, the coherence of the sampling matrix in the DCT basis of $\mu^{\mathrm{DCT}}=0.2$ is accomplished with $M=570$ measurements. Whereas in the multipixel case, for $l=16$, the same $\mu^{\mathrm{DCT}}$ is achievable (at $\sigma=1.3$ ) for $M=6$ measurements. This is a reduction of factor 100 at the cost of $l^{2}=256$ more detectors.

The reduction of necessary measurements for sparse signals in the DCT basis works well, but as Figs. 3(d) and 4(d) show, it does not work in the (Haar) wavelet domain. The reason for this is the local selectivity of each single detector and the associated problems with the wavelet transform we discussed above. This is the reason why we use only the DCT domain in our experiments.

Another very interesting aspect is the best value for $\sigma$ in the multidetector case. In Fig. 4(b), it is obvious that there is some optimal value for $\sigma$. If $\sigma$ is too large, each detector more or less "sees" the same blurred image, and on the other hand, if $\sigma$ is very low, the response is concentrated to very few local pixels in the high-resolution image. Fig. 4(b) indicates an optimal value of $\sigma \approx 1$ that is supported by other experiments we did, which all indicate an optimal value of $\sigma=N_{u} / 2 l=N_{v} / 2 l$ for the rectangular case $\left(N_{u}=N_{v}\right)$. The consequence is that system performance is at its best if the detector array is in focus. Even with the perfect focus, we cannot achieve optimal blurring because of the size of each detector.

In Table I, we present the system performance in terms of peak signal-to-noise ratio (PSNR) using four images from the Berkeley segmentation database [23]. ${ }^{1}$ The images were changed to gray scale and then resized to [32 $\times 32]$ pixels before they were used as ground truth. For reference purpose, the PSNR of the best $K$-sparse approximation in the DCT basis is calculated for each image and displayed in the table header. All other PSNR readings in the table represent the quality of a $K$-sparse (with $K=30$ nonzero elements) reconstruction from measurements taken with respective model parameters $l$ and $M$. Reconstruction was performed in the DCT basis with compressive sampling matching pursuit (CoSaMP) [24], which is a fast greedy algorithm to solve (3), where it is guaranteed that the solution is exactly $K$-sparse. Blurring intensity was set to $\sigma=32 / 2 l$. It can be seen how the number of sequent measurements can be decreased if more detectors are used.

\section{EXPERIMENTS}

Here, we present some results of the GC, point-spread function (PSF) estimation, simulations of the calibrated model, and $\mathrm{CS}$ reconstructions.

\footnotetext{
${ }^{1}$ http://www.eecs.berkeley.edu/Research/Projects/CS/vision/grouping/ segbench/BSDS300-images.tgz
} 
TABLE I

RECONSTRUCTION QUALITY USING DiFFERENT NUMBER OF DETECTORS $\left(L=l^{2}\right)$ AND DifFERENT Number of SEQUent Measurements $(M)$. Simulated With Four Images From the Berkeley SEgMENTATION Database [21]. All Images Were Scaled to [32 × 32] PiXels, and $K=30$ NonZERo COEFFICIENTS WERE RECONSTRUCTED IN THE DCT BASE With THE COSAMP ALGORITHM

\begin{tabular}{|c|c|c|c|c|}
\hline \multirow{2}{*}{ Dovit } & \multicolumn{4}{|c|}{$\begin{array}{l}\text { Filename: } 100075 \cdot j \mathrm{jpg} \\
\text { K-Sparse approximation: } 23.87 \mathrm{~dB}\end{array}$} \\
\hline & $L=1$ & $L=4$ & $L=16$ & $L=256$ \\
\hline$M=3$ & $8.11 \mathrm{~dB}$ & $8.06 \mathrm{~dB}$ & $7.68 \mathrm{~dB}$ & $16.47 \mathrm{~dB}$ \\
\hline$M=30$ & $8.93 \mathrm{~dB}$ & $10.41 \mathrm{~dB}$ & $15.43 \mathrm{~dB}$ & $76 \mathrm{~dB}$ \\
\hline$M=300$ & $20.66 \mathrm{~dB}$ & $21.07 \mathrm{~dB}$ & $23.21 \mathrm{~dB}$ & $23.37 \mathrm{~dB}$ \\
\hline \multirow[b]{2}{*}{ r... } & \multicolumn{4}{|c|}{$\begin{array}{l}\text { Filename: } 161062 \cdot j \mathrm{jpg} \\
\text { K-Sparse approximation: } 28.46 \mathrm{~dB}\end{array}$} \\
\hline & $L=1$ & $L=4$ & $L=16$ & $L=256$ \\
\hline$M=3$ & $4.14 \mathrm{~dB}$ & $3.73 \mathrm{~dB}$ & $3.27 \mathrm{~dB}$ & $19.28 \mathrm{~dB}$ \\
\hline$M=30$ & $4.67 \mathrm{~dB}$ & $7.32 \mathrm{~dB}$ & $20.47 \mathrm{~dB}$ & $8 \mathrm{~dB}$ \\
\hline$M=300$ & $25.65 \mathrm{~dB}$ & $25.96 \mathrm{~dB}$ & $27.53 \mathrm{~dB}$ & $4 \mathrm{~dB}$ \\
\hline & \multicolumn{4}{|c|}{$\begin{array}{l}\text { Filename: } 220075 \cdot \mathrm{jpg} \\
\text { K-Sparse approximation: } 19.97 \mathrm{~dB}\end{array}$} \\
\hline & $L=1$ & $L=4$ & $L=16$ & $L=256$ \\
\hline$M=3$ & $8.18 \mathrm{~dB}$ & $8.07 \mathrm{~dB}$ & $7.83 \mathrm{~dB}$ & $12.47 \mathrm{~dB}$ \\
\hline$M=30$ & $8.74 \mathrm{~dB}$ & $10.46 \mathrm{~dB}$ & $12.62 \mathrm{~dB}$ & $18.58 \mathrm{~dB}$ \\
\hline$M=300$ & $17.17 \mathrm{~dB}$ & $17.00 \mathrm{~dB}$ & $18.71 \mathrm{~dB}$ & $19.76 \mathrm{~dB}$ \\
\hline & \multicolumn{4}{|c|}{$\begin{array}{l}\text { Filename: } 253027 \cdot j \mathrm{jpg} \\
\text { K-Sparse approximation: } 26.24 \mathrm{~dB}\end{array}$} \\
\hline & $L=1$ & $L=4$ & $L=16$ & $L=256$ \\
\hline$M=3$ & $6.86 \mathrm{~dB}$ & $6.25 \mathrm{~dB}$ & $7.73 \mathrm{~dB}$ & $17.03 \mathrm{~dB}$ \\
\hline$M=30$ & $7.40 \mathrm{~dB}$ & $9.38 \mathrm{~dB}$ & $18.02 \mathrm{~dB}$ & $25.02 \mathrm{~dB}$ \\
\hline$M=300$ & $23.41 \mathrm{~dB}$ & $23.31 \mathrm{~dB}$ & $25.07 \mathrm{~dB}$ & $26.03 \mathrm{~dB}$ \\
\hline
\end{tabular}

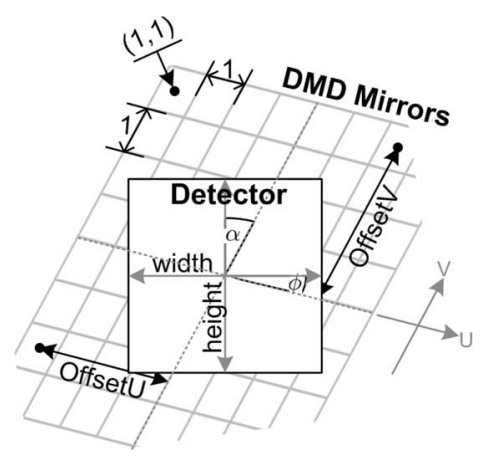

Fig. 5. Schematic of a single-pixel camera's position in relation to the mirrors of the DMD under affine projection.

\section{A. $G C$}

With GC, the detector-to-mirror relationship is determined. For calibration, we use as many circular control points as possible to fit on the DMD array with a resolution of $\left[N_{u} \times N_{v}\right]=$ $[1024 \times 768]$. For a good calibration, it is necessary to have many large control points. In Fig. 6, the repetition accuracy of different combinations for a number of control points and their size is displayed. In the figure, we have calibrate the system 100 times for every combination and calculated the standard deviation (given in mirror dimensions) of a reference-detector's offset (see Fig. 5). As a reference detector, we used the one closest to the top left, center, and bottom right mirror locations. In Fig. 6, we see a very good repetition accuracy value of about 0.1 times a mirror size. In the given setup, each detector had width and height of 3.44 and 3.52 in mirror dimensions, respectively.

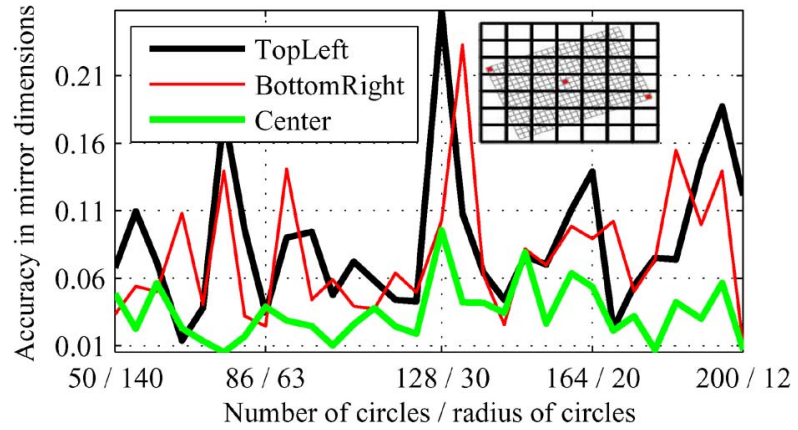

Fig. 6. Location offset repetition accuracy values of three detectors, which are associated with the top left, center, and bottom right mirrors of the DMD (see Fig. 5). Accuracy is measured as standard deviation over 100 calibrations at different number of control points and different radii of these points. (a)

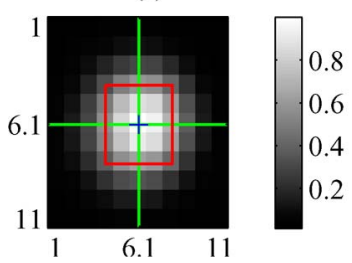

(b)

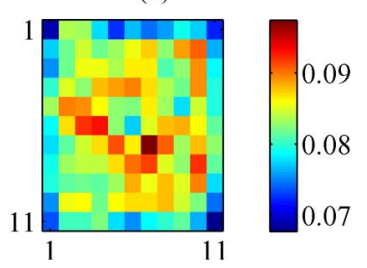

Fig. 7. PSF of system. (a) Mean over 10 measurements with different random binary DMD patterns and PSF estimations using an area of [700 $\times 500]$ mirrors. The cross is located at the point of gravity, and the square indicates the size of a single detector. (b) Standard deviation of each element in the PSF.

\section{B. Blur Estimation}

To measure blurring, which is defined by its PSF of the setup, we solved (18) for an impulse response with size $[11 \times 11]$ and a random binary DMD pattern $\mathbf{F}_{k}$. In Fig. 7, the result of the estimation is shown. Fig. 7(a) presents the mean PSF over 10 measurements, and in Fig. 7(b), the standard deviation of each element in the PSF is displayed. The PSF estimation, as well as GC, tries to find out the relationship of mirrors to detectors on the array. While GC operates on a large scale, PSF estimation considers only mirrors around the calibrated center of a detector. The PSF we estimated for our setup [see Fig. 7(a)] is well centered and thus indicates a very precise subpixel GC because the detectors are symmetrically influenced by "underlying" mirrors.

The relatively high standard deviation of the PSF elements is caused by inhomogeneity of the scene during calibration. To compensate for this, we used many measurements with different DMD patterns and a large area of [168 $\times 210]$ mirrors.

\section{Simulations Using the Calibrated Model}

With the calibrated model, we simulate the system and show its theoretical super-resolution performance. In Fig. 8, two ground truth images (a) and (d) were sampled $M$ times with the calibrated model according to (2). The resolution of the detector array is set to $\left[N_{x} \times N_{y}\right]=[16 \times 23]$, and the resolution of the reconstructed image is $\left[N_{u} \times N_{v}\right]=[55 \times 81]$. The scaling between low- and high-resolution pixels is $\left(S_{1}, S_{2}\right)=$ $(3.44,3.52)$ [see (2)]. In Fig. 8(b) and (e), the output of the detector array is shown without any multiplication pattern $\mathbf{F}_{k}$ applied. This is the best resolution one could expect from the 
(a)

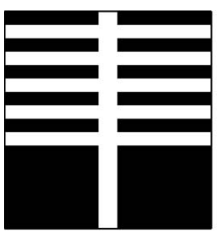

(d)

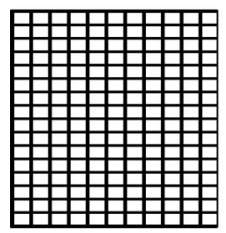

(b)

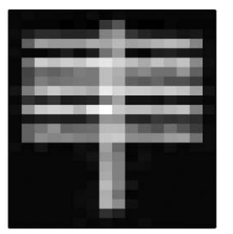

(e)

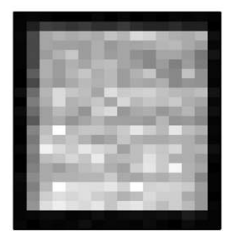

(c)

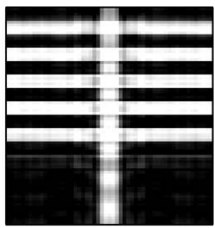

(f)

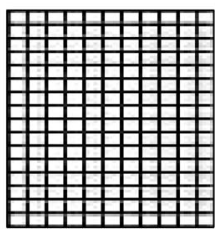

Fig. 8. Reconstruction of simulated measurements with the calibrated model. [(a) and (d)] Original images $([55 \times 81]$ pixels). [(b) and $(e)]$ Low-resolution output of detector array $([16 \times 23]$ detectors $)$ without any multiplication pattern $\mathbf{F}_{k}$ applied. (c) Reconstruction using $M=10$ measurements and $K=50$ in DCT basis. The best $K$-sparse approximation is $17.96 \mathrm{~dB}$, and the reconstruction result is $17.77 \mathrm{~dB}$. (f) Reconstruction result. The best $K$-sparse approximation is $19.62 \mathrm{~dB}$, and the reconstruction result is $19.53 \mathrm{~dB}$.

system without the discussed approach. In Fig. 8(c) and (f), the reconstruction result using the calibrated model with $M=$ 10 is presented. $K$-sparse reconstruction was performed with $K=50$ in the DCT basis with the CoSaMP algorithm. The reconstructions show the possible super-resolution performance of the calibrated model. In particular, the bottom image (d) is reconstructed well because of its periodic structure, which can be approximated very sparsly in the DCT basis.

\section{Real Data Measurements}

With the measurement setup discussed in Section II, we took sampling data and reconstructed them using the same calibrated model that was used for the simulation above and the CoSaMP algorithm. Reconstruction was performed in the DCT basis, and resolution of the reconstructed image and low-resolution measurement were $[55 \times 81]$ pixels and $[16 \times 23]$ detectors, respectively.

Fig. 9(c) shows the reconstruction of a periodic structure, which can be represented very sparsely in the DCT basis. Fig. 9(a) is a picture of the scene, and in Fig. 9(b), the camera (detector array) image is presented. Using only $M=20$ measurements and $K=30$, we were able to reconstruct the image in Fig. 9(c). The reconstruction of a second object is shown in Fig. 9(f). A picture of this object is presented in Fig. 9(d). Obviously, its representation in the DCT basis is not as sparse as that in the last example. Thus, we used $K=300$ and $M=$ 30 measurements to reconstruct a high-resolution image. The detector array image is shown in Fig. 9(e). In general, these real experiments show the super-resolution performance of our setup and our new model as the reconstructed images have a far better resolution compared to the detector array on its own.

\section{CONCLUSION}

In this paper, we have presented a new CS model and a realworld test setup covered by this model. We presented methods for successfully calibrating the model with all its parameters (a)

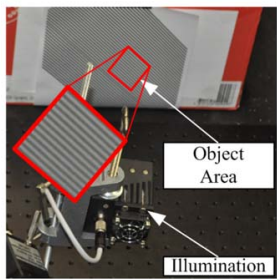

(d)

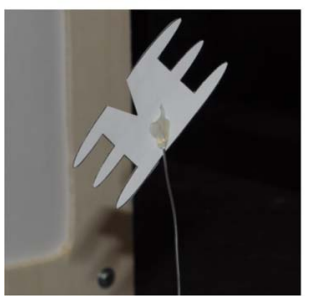

(b)

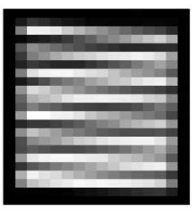

(e)

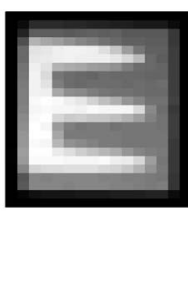

(c)

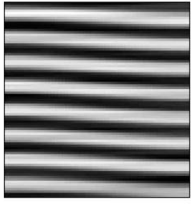

(f)

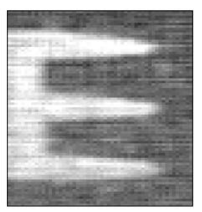

Fig. 9. Reconstruction results of real data. [(a) and (d)] Picture taken of the scene. [(b) and (e)] Best image observable by the detector array. [(c) and (f)] Reconstruction of the scene using $M=20$ and $M=30$ measurements. The upscaling factors are $\left(S_{1}, S_{2}\right)=(3.44,3.52)$.

to the test setup. Measurement results regarding the calibration accuracy are presented. We also provided quality measures of the sampling model for different model parameters based on well-established quality measures. A variety of simulations were presented on different model parameters and on an actual calibrated model to show the performance of our new model. In addition to this, we presented the reconstruction of real measurements performed with the proposed setup.

From all our experiments, it is clear that, if multiple detectors can be used, there is a benefit compared with a single detector in terms of measurement time and number of sequent measurements while providing comparable reconstruction quality.

\section{REFERENCES}

[1] M. F. Duarte, M. A. Davenport, D. Takhar, J. N. Laska, T. Sun, K. F. Kelly, and R. G. Baraniuk, "Single-pixel imaging via compressive sampling," IEEE Signal Process. Mag., vol. 25, no. 2, pp. 83-91, Mar. 2008.

[2] A. Heidari and D. Saeedkia, "A 2D camera design with a single-pixel detector," in Proc. 34th Int. Conf. Citeseer IRMMW-THz, 2009, pp. 1-2.

[3] W. L. Chan, K. Charan, D. Takhar, K. F. Kelly, R. G. Baraniuk, and D. M. Mittleman, "A single-pixel terahertz imaging system based on compressed sensing," Appl. Phys. Lett., vol. 93, no. 12, p. 121 105, Sep. 2009.

[4] J. Yang, J. Wright, T. Huang, and Y. Ma, "Image super-resolution as sparse representation of raw image patches," in Proc. CVPR, 2008, pp. 1-8.

[5] A. Griffin and P. Tsakalides, "Compressed sensing of audio signals using multiple sensors," Reconstruction, vol. 3, no. 4, p. 5, 2005.

[6] D. Baron, M. B. Wakin, M. F. Duarte, S. Sarvotham, and R. G. Baraniuk, "Distributed compressed sensing," 2005, to be published.

[7] M. Lustig, D. Donoho, and J. M. Pauly, "Sparse MRI: The application of compressed sensing for rapid MR imaging," Magn. Reson. Med., vol. 58, no. 6, pp. 1182-1195, Dec. 2007.

[8] J. Ma and M. Y. Hussaini, "Extensions of compressed imaging: Flying sensor, coded mask, and fast decoding," IEEE Trans. Instrum. Meas., vol. 60, no. 9, pp. 3128-3139, Sep. 2011.

[9] B. K. Natarajan, "Sparse approximate solutions to linear systems," SIAM J. Comput., vol. 24, no. 2, pp. 227-234, Apr. 1995.

[10] D. L. Donoho, "Compressed sensing," IEEE Trans. Inf. Theory, vol. 52, no. 4, pp. 1289-1306, Apr. 2006.

[11] E. J. Candès and T. Tao, "Decoding by linear programming," IEEE Trans. Inf. Theory, vol. 51, no. 12, pp. 4203-4215, Dec. 2005.

[12] J. Ma and F. X. Le Dimet, "Deblurring from highly incomplete measurements for remote sensing," IEEE Trans. Geosci. Remote Sens., vol. 47, no. 3, pp. 792-802, Mar. 2009 
[13] T. Edeler, S. Hussmann, K. Ohliger, and A. Mertins, "Parameter calibration of a novel super-resolution model for a compressed-sensing measurement setup," in Proc. I2MTC, May 2011, pp. 1376-1380.

[14] R. Hartley and A. Zisserman, Multiple View Geometry in Computer Vision. Cambridge, U.K.: Cambridge Univ. Press, 2003.

[15] J. Heikkila, "Geometric camera calibration using circular control points," IEEE Trans. Pattern Anal. Mach. Intell., vol. 22, no. 10, pp. 1066-1077, Oct. 2000.

[16] E. J. Candès, "The restricted isometry property and its implications for compressed sensing," Comptes Rendus Math., vol. 346, no. 9-10, pp. 589-592, 2008.

[17] J. D. Blanchard, C. Cartis, J. Tanner, and A. Thompson, "Phase transitions for greedy sparse approximation algorithms," Appl. Comput. Harmonic Anal., vol. 30, no. 2, pp. 188-203, Mar. 2011, ISSN 1063-5203, 10.1016/j.acha.2010.07.001.

[18] N. Vaswani and W. Lu, "Modified-CS: Modifying compressive sensing for problems with partially known support," IEEE Trans. Signal Process., vol. 58, no. 9, pp. 4595-4607, Sep. 2010.

[19] C. Dossal, G. Peyrè, and J. Fadili, "A numerical exploration of compressed sampling recovery," Linear Algebra Appl., vol. 432, no. 7, pp. 1663-1679, Mar. 2010.

[20] D. L. Donoho and X. Huo, "Uncertainty principles and ideal atomic decomposition," IEEE Trans. Inf. Theory, vol. 47, no. 7, pp. 2845-2862, Nov. 2001

[21] D. L. Donoho, M. Elad, and V. N. Temlyakov, "Stable recovery of sparse overcomplete representations in the presence of noise," IEEE Trans. Inf. Theory, vol. 52, no. 1, pp. 6-18, Jan. 2006.

[22] H. Rauhut, K. Schnass, and P. Vandergheynst, "Compressed sensing and redundant dictionaries," IEEE Trans. Inf. Theory, vol. 54, no. 5, pp. 2210 2219, May 2008.

[23] D. Martin, C. Fowlkes, D. Tal, and J. Malik, "A database of human segmented natural images and its application to evaluating segmentation algorithms and measuring ecological statistics," in Proc. 8th Int. Conf. Comput. Vis., Jul. 2001, vol. 2, pp. 416-423.

[24] D. Needell and J. A. Tropp, "CoSaMP: Iterative signal recovery from incomplete and inaccurate samples," Appl. Comput. Harmonic Anal., vol. 26, no. 3, pp. 301-321, 2009.

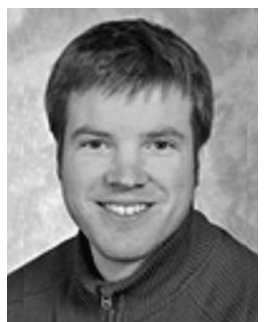

Torsten Edeler received the Dipl.-Ing. (FH) degree from Hamburg University of Applied Sciences (HAW), Hamburg, Germany, in 2004. He is currently working toward the Ph.D. degree at Westcoast University of Applied Sciences (FHW), Heide, Germany.

From 2004 to 2007 , he gained industrial experience as an Electronic Development Engineer at one of the leading manufacturers of machine vision cameras. His research interests include signal reconstruction by exploiting sparse representation, super resolution, noise reduction, and algorithm design for field-programmable gate array implementation.

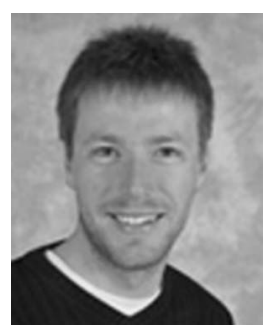

Kevin Ohliger received the Dipl.-Ing. (FH) degree in 2005 from Westcoast University of Applied Sciences (FHW), Heide, Germany, where he is currently working toward the Ph.D. degree.

From 2005 to 2007, he gained industrial experience as an Electronic Development Engineer at one of the leading manufacturers of aircraft electronics. His research interests include segmentation and active contours.

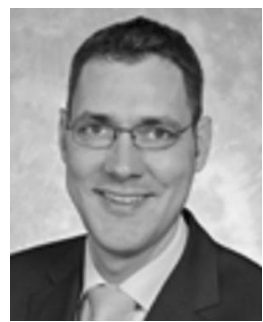

Stephan Hussmann (SM'11) received the M.E. and $\mathrm{Ph}$.D. degrees from the University of Siegen, Siegen, Germany, in 1995 and 2000, respectively.

From 1996 to 2000, he was a Research Associate with the Center for Sensor Systems (ZESS), University of Siegen, and a Development Engineer with Aicoss GmbH, Siegen. From 2001 to 2003, he was a Lecturer with the Department of Electrical and Computer Engineering, University of Auckland, Auckland, New Zealand. Since the end of 2004, he has been a Professor with the Department of Electrical Engineering and Information Technologies, Westcoast University of Applied Sciences (FHW), Heide, Germany, in the area of microprocessor technology and electronic systems. He has widely consulted with the industry and has more than 60 publications, which include book chapters, international patents, and refereed journal and conference proceeding papers. His research interests include low-cost multisensor system design, real-time 2-D/3-D image processing, embedded systems design, and engineering education.

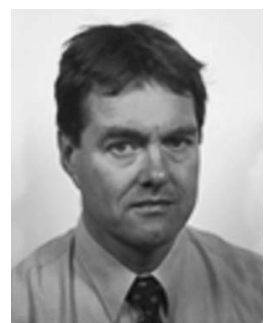

Alfred Mertins (M'96-SM'03) received the Dipl.-Ing. degree from the University of Paderborn, Paderborn, Germany, in 1984, the Dr.-Ing. degree in electrical engineering and the Dr.-Ing.habil. degree in telecommunications from Hamburg University of Technology, Hamburg, Germany, in 1991 and 1994. respectively

From 1986 to 1991, he was a Research Assistant with Hamburg University of Technology, and from 1991 to 1995 , he was a Senior Scientist with the Microelectronics Applications Center Hamburg. From 1996 to 1997, he was with the University of Kiel, Kiel, Germany, and from 1997 to 1998 , he was with the University of Western Australia, Perth, Australia. In 1998, he joined the University of Wollongong, Wollongong, Australia, where he was an Associate Professor of electrical engineering. From 2003 to 2006, he was a Professor with the Faculty of Mathematics and Science, University of Oldenburg, Oldenburg, Germany. In November 2006, he joined the University of Lübeck, Lübeck, Germany, where he is currently a Professor and the Director of the Institute for Signal Processing. His research interests include speech, audio, and image processing; wavelets and filter banks; pattern recognition; and digital communications. 